\title{
Is left ventricular systolic index the new kid in the block?
}

\author{
Vita Tomas, MD, MPH, a and Vita Nestor, MD, FACC ${ }^{\mathrm{b}, \mathrm{c}}$ \\ a Cardiovascular Imaging Program, Departments of Medicine (Cardiovascular Division) and \\ Radiology, Brigham and Women's Hospital, Boston \\ b Cardiovascular Division, Italian Hospital of Rosario, Rosario, Argentina \\ c Cardiovascular Instituto of Rosario “Dr. González Sabathie”, Rosario, Argentina
}

Received Nov 29, 2017; accepted Nov 30, 2017

doi: $10.1007 / \mathrm{s} 12350-017-1170-y$

\section{See related article, pp. 1148-1156}

There are many diagnostic and prognostic elements arising from myocardial perfusion imaging studies. The equipment improvements have reduced acquisition time while enhancing quality imaging. In line with technological developments, a greater understanding of coronary pathophysiology, especially microcirculation, has allowed the clinical cardiologist to understand coronary artery disease (CAD). In the past, many cases had no physiopathology correlation with the clinical setting, for example, angina with normal coronary arteries. Nowadays, we have a more clear view of such findings.

From the very beginning, ventricular dilatation (VD) during stress has proven to be associated with adverse outcomes, considering secondary to extensive ischemia.

The prognostic value of VD has been associated with the presence of severe and extensive coronary disease since Chouraqui et al. ${ }^{1}$ and Weiss et al. ${ }^{2}$ publications 30 years ago.

Exploring further, Mazzanti et al. ${ }^{3}$ correlated the ischemia severity with VD using cinecoronariography as the gold standard. They showed a 71 and $95 \%$ of

Reprint requests: Vita Nestor, MD, FACC, Cardiovascular Division, Italian Hospital of Rosario, Rosario, Argentina, nestor.vita@ gmail.com

J Nucl Cardiol 2019;26:1157-60.

1071-3581/\$34.00

Copyright (C) 2018 American Society of Nuclear Cardiology. sensibility and specificity, respectively, by semiquantitative analysis.

Ventricular dilatation without ischemia and normal myocardial perfusion imaging (MPI) have a less clear clinical and prognostic value. Gated SPECT (gSPECT) imaging relies on detection of endocardial edge for measurements of left ventricle (LV) volumes and transient ischemic dilatation (TID) ratio. The TID threshold depends on the radioisotope used, stress methods, proper algorithm, the avoidance of artifact motion, incorrect slice alignment, and the clinical setting of the population studied. Different cutoffs have been validated for different radioisotope and stress protocols (Table 1). ${ }^{4}$

There are two possible interpretations related to VD in the gSPECT. The classic understanding is that there is extensive subendocardial ischemia generating stunning. The second interpretation, which is more frequently seen with pharmacological stress than exercise, is that adenosine induces subendocardial hypoperfusion. In patients with limited coronary flow reserve (CFR), adenosine produces vasodilatation in the vascular bed of both, the epicardial and endocardial vessels. As a consequence, there is an increasing flow from the subendocardium to the epicardium. This is different from ischemia in CAD. We called it "vertical steal", and it is a marker of impaired subendocardial CFR rather than a true myocardial stunning. The "vertical steal" explains the increasing frequency of TID in patients with diseases such as diabetes and hypertension and could go along with a change in left ventricular remodeling, more significant with adenosine than with exercise.

Emmett et al. ${ }^{5}$ studied TID on myocardial perfusion imaging with pharmacological stress. The author evaluated if TID was due to actual LV cavity dilation and ventricular stunning or to relative subendocardial hypoperfusion. He studied 31 patients undergoing 
Tc99 $\mathrm{m}$ adenosine sestamibi MPI and transthoracic echocardiogram acquired at rest, immediately post stress and $2 \mathrm{~h}$ later. While they found end-diastolic volume and end-systolic volume dilatation in the nuclear studies, these changes were not seen with post stress echocardiography. In the TID (+) group, there was no LV dilation or changes in volumes by echocardiography. This increases the possibility that global reduction in perfusion at the endocardial border led to an apparent increase in end-systolic volume.

As PET developed, a better physiopathological understanding was achievable. Using CFR allowed the evaluation of significant changes in coronary flow, detecting balance ischemia - severe CAD—in subjects with normal MPI and TID. This is a reliable and valid method to assess subendocardial ischemia.

In addition to TID diagnostic values, Abidov et al. ${ }^{6}$ showed its prognostic value in patients with normal MPI. Compared to patients without TID those in the highest quartile with VD showed a threefold increase in annualized event rate $(2.4 \%$ vs. $0.8 \% ; p=0.001)$. Similarly, Doukky et al. ${ }^{7}$ studied 294 patients with diabetes mellitus or CAD. The study subjects were followed for $27 \pm 9$ months for cardiac death or myocardial infarction (MI). Patients with TID had an annual event rate of $13.3 \%$ versus $0.4 \%(p=0.001)$ in those without TID.

\section{REMODELING}

Braunwald $^{8}$ and Meizlish ${ }^{9}$ decades ago showed an increase in major cardiac events in patients with acute MI or advance heart failure when the LV lost the ellipsoidal shape and increased its volume regardless of LVEF. Similar to LV volume, cardiac remodeling could be related to an increase in adverse events in the right clinical scenario.

Nuclear cardiology techniques have many toolsLVEF, TID, CFR among others-to determine patients diagnosis and prognosis. In this context, LV shape change measured as LV shape index (LVSI) could add a new prognostic tool of difficult independent assessment. LVSI is a quantitative 3-dimensional index. It is defined as the ratio of the maximum short-axis dimension of the LV cavity to the long-axis dimension, from the endocardial apex to the center of the plane valve (Figure. 1). ${ }^{10}$ Ratio values closer to 1 refer to a more spherical shape. On the other hand, values towards 0 relate to an ellipsoidal shape.

In this edition, Gaudieri et al. ${ }^{13}$ studied the prognostic value of LVSI in 646 patients with normal myocardial perfusion and normal LVEF on stress-gated SPECT imaging and follow-up for a median of 37 months. They observed a significantly higher number 


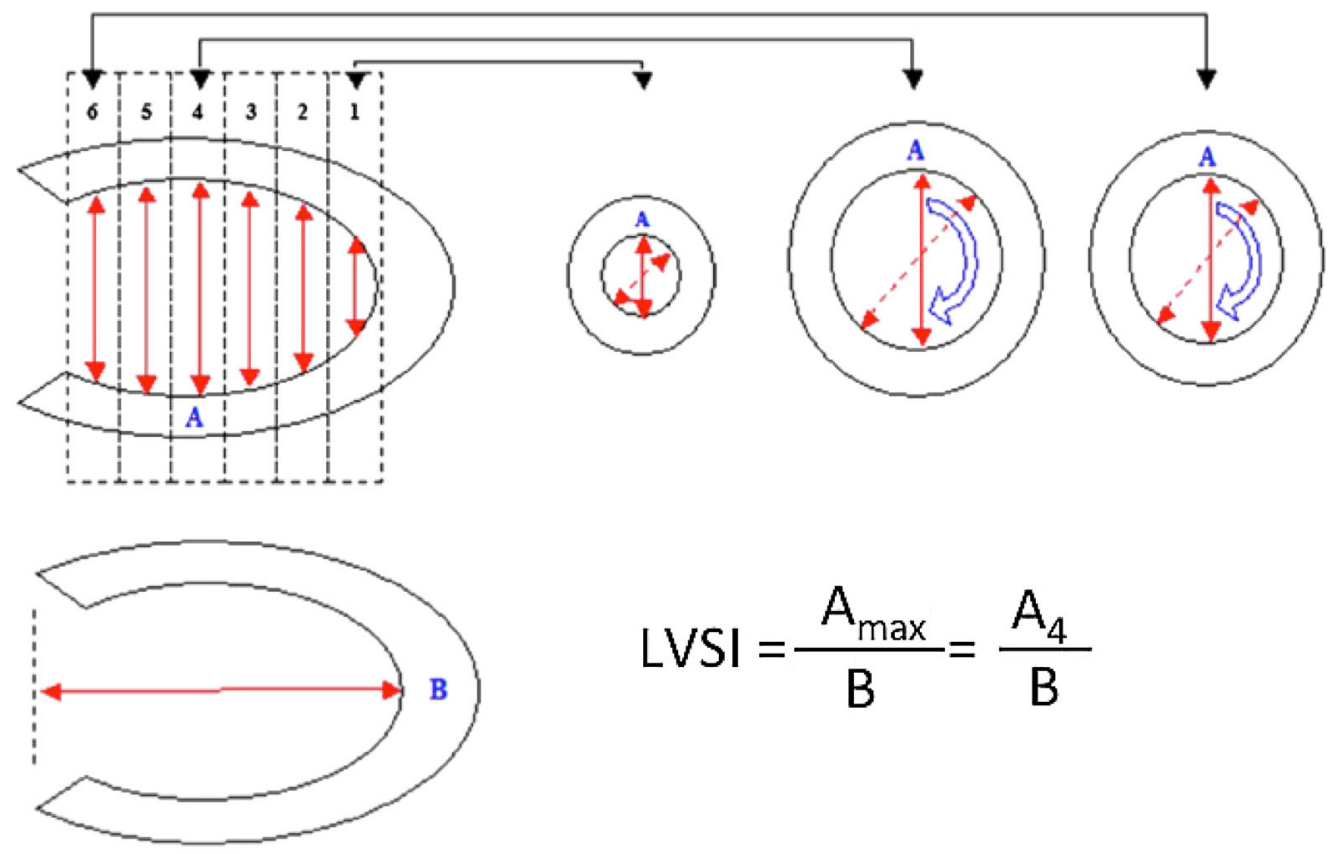

\section{In this figure $A_{\max }$ is represented by the short axis in slice $4\left(A_{4}\right)$}

Figure 1. Left ventricular systolic index is calculated as the ratio of maximum transverse dimension $\left(\mathrm{A}_{\max }\right)$ to maximum longitudinal dimension $(\mathrm{B})$.

of adverse events-cardiac death, non-fatal myocardial infarction, or unstable angina requiring coronary revascularization-in patients with an LVSI $>0.54$, which remains significant when adjusted for age and diabetes. Interestingly, the authors were evaluating the value of LVSI as a cardiac remodeling marker but they did not include HF hospitalizations as an outcome. Also, half of the events in this study occurred in the adenosine stress subgroup, which represents $40 \%$ of the cohort. Although pharmacological stress test was not significant in the univariable analysis, these patients could have driven the results. As mentioned previously, adenosine produces "vertical steal," which is seen as TID and it could also have caused an increase of LVSI without showing true remodeling. As patients studied with adenosine tend to be iller-older, higher prevalence of diabetes, and lower functional capacity-, this could have driven LVSI association with adverse events.

It would be worth to see (a) TID significance in the analysis, (b) the percentage of patients with LVSI in the pharmacological stress subgroup and in the exercise stress group, and (c) HF hospitalization as an end point.

While LVSI is emerging as a new parameter and eventually a prognostic marker, ${ }^{10-12}$ there are still several interrogates to consider its implementation. Does LVSI provide better information than other wellknown and straightforward markers such as LVEF or
TID? Is LVSI reproducible? What is the applicability of LVSI?

Interestingly, in Gaudieri et al.'s ${ }^{13}$ work, one of the most robust prognostic markers, LVEF, was not significantly associated with the endpoints due to inclusion criteria. Being tested in a homogeneous cohort with normal LVEF, LVSI could evaporate its prognostic value in more heterogeneous populations. It would be interesting to evaluate LVSI in a model which includes LVEF and TID as well as evaluate LVSI and TID correlation.

As mentioned previously, another commonly used marker is TID. While LVSI could have an advantage over TID-since it provides a 3-dimensional information-, LVSI could face some challenges on generalization: unclear cutoff for different protocols, machines, and populations and various possible physiological processes represented with the same image pattern. As TID's most reliable cutoff is 2SD from the normal population, LVSI needs to establish reference pattern to be disseminated. Unfortunately, there is evidence $^{12}$ that shows that dissemination would not be possible across different stress protocols. Another relevant aspect is LVSI reproducibility. Although authors affirm it is a robust and easy to reproduce measure, no method is free from operator influence. In those with perfusion defects, measuring axes and ultimately LVSI 
may prove difficult and inaccurate. SPECT has a low spatial resolution. A small displacement of the limits would change LVSI values dramatically, which could affect in-center and out-center reproducibility. Also, it would be interesting to see how this software is used and interpreted when there is no "software expert"' in place.

TID is ingrained and well understood by imaging clinician. In this context, LVSI will have to find its fit in everyday workflow. Similar to how glycemia and glycosylated hemoglobin are used, TID could represent acute and short-term injury to oxygen misbalance (stunning), while LVSI could possibly be interpreted as the remodeling response to chronic-sustained deleterious factors.

From a physiopathological perspective, LVSI sounds as a simple and logic marker. However, until more data are collected these authors cannot recommend its use in everyday practice. We could imagine that positive LVSI (LVSI > 2SD) in exercise stress test will have analogous use to TID in clinical practice. On one hand, based on previous studies, ${ }^{11}$ in patients with normal MPI and risk factors, such as patients with diabetes, history of CAD, or chronic kidney disease, further tests should be requested-Calcium score, stress MRI, or echo. On the other hand, in patients with abnormal MPI suggesting CAD, a positive LVSI should raise concerns and it would be reasonable to progress with a method that shows coronary anatomy and eventually the fractional flow reserve.

\section{Disclosure}

$V$. Tomas and V. Nestor authors have indicated that they have no financial conflict of interest.

\section{References}

1. Chouraqui P, Rodrigues EA, Berman DS, Maddahi J. Significance of dipyridamole-induced transient dilation of the left ventricle during thallium-201 scintigraphy in suspected coronary artery disease. Am J Cardiol. 1990;66:689-94.

2. Weiss AT, Berman DS, Lew AS, Nielsen J, Potkin B, Swan HJ, Waxman A, Maddahi J. Transient ischemic dilation of the left ventricle on stress thallium-201 scintigraphy: a marker of severe and extensive coronary artery disease. J Am Coll Cardiol. 1987;9:752-9.
3. Mazzanti M, Germano G, Kiat H, Kavanagh PB, Alexanderson E, Friedman JD, Hachamovitch R, Van Train KF, Berman DS. Identification of severe and extensive coronary artery disease by automatic measurement of transient ischemic dilation of the left ventricle in dual-isotope myocardial perfusion SPECT. J Am Coll Cardiol. 1996;27:1612-20.

4. Slomka PJ, Berman DS, Germano G. Normal limits for transient ischemic dilation with $99 \mathrm{mTc}$ myocardial perfusion SPECT protocols. J Nucl Cardiol. 2017;24:1709-11.

5. Emmett L, Ng A, Ha L, Russo R, Mansberg R, Zhao W, Chow SV, Kritharides L. Comparative assessment of rest and post-stress left ventricular volumes and left ventricular ejection fraction on gated myocardial perfusion imaging (MPI) and echocardiography in patients with transient ischaemic dilation on adenosine MPI: myocardial stunning or subendocardial hypoperfusion? J Nucl Cardiol. 2012;19:735-42.

6. Abidov A, Bax JJ, Hayes SW, Hachamovitch R, Cohen I, Gerlach J, Kang X, Friedman JD, Germano G, Berman DS. Transient ischemic dilation ratio of the left ventricle is a significant predictor of future cardiac events in patients with otherwise normal myocardial perfusion SPECT. J Am Coll Cardiol. 2003;42:181825 .

7. Doukky R, Frogge N, Bayissa YA, Balakrishnan G, Skelton JM, Confer K, Parikh K, Kelly RF. The prognostic value of transient ischemic dilatation with otherwise normal SPECT myocardial perfusion imaging: a cautionary note in patients with diabetes and coronary artery disease. J Nucl Cardiol. 2013;20:774-84.

8. Pfeffer MA, Braunwald E. Ventricular remodeling after myocardial infarction. Experimental observations and clinical implications. Circulation. 1990;81:1161-72.

9. Meizlish JL, Berger HJ, Plankey M, Errico D, Levy W, Zaret BL. Functional left ventricular aneurysm formation after acute anterior transmural myocardial infarction. Incidence, natural history, and prognostic implications. N Engl J Med. 1984;311:1001-6.

10. Abidov A, Slomka PJ, Nishina H, Hayes SW, Kang X, Yoda S, Yang LD, Gerlach J, Aboul-Enein F, Cohen I, Friedman JD, Kavanagh PB, Germano G, Berman DS. Left ventricular shape index assessed by gated stress myocardial perfusion SPECT: initial description of a new variable. J Nucl Cardiol. 2006;13:652-9.

11. Nappi C, Gaudieri V, Acampa W, Assante R, Zampella E, Mainolfi CG, Petretta M, Germano G, Cuocolo A. Comparison of left ventricular shape by gated SPECT imaging in diabetic and nondiabetic patients with normal myocardial perfusion: a propensity score analysis. J Nucl Cardiol. 2017. https:// doi.org/10.1007/s12350-017-1009-6.

12. Gimelli A, Liga R, Giorgetti A, Casagranda M, Marzullo P. Stressinduced alteration of left ventricular eccentricity: an additional marker of multivessel CAD. J Nucl Cardiol. 2017. https:// doi.org/10.1007/s12350-017-0862-7.

13. Gaudieri V, Nappi C, Acampa W, Zampella E, Assante R, Mannarino T, Genova A, De Simini G, Klain M, Germano G, Petretta M, Cuocolo A. Added prognostic value of left ventricular shape by gated SPECT imaging in patients with suspected coronary artery disease and normal myocardial perfusion. J Nucl Cardiol. 2017. https://doi.org/10.1007/s12350-017-1090-x. 